least 3 times. Directly measured health variables were not associated with number of residential moves made at 18 or 36 . Odds of scoring at least 3 on the GHO12 questionnaire were significantly increased at age 18 for those moving 1-2 times (OR 2.01 (1.36-2.96)) and those moving 3 times or more (OR $2.04(1.3,3.22)$ ) compared to those who remained stable. Similarly, odds of reporting a longstanding illness at 18 were increased for $1-2$ moves (OR 1.88 (1.11, $3.18)$ ) and at least 3 moves $(\mathrm{OR}=2.03(1.11,3.69))$. Odds were elevated, but not significant, at 36 for these health variables. Odds of trying drugs and smoking at 18 were significantly increased but only for those moving at least 3 times. Although elevated, odds for these health behaviours were not significant at 36 .

Conclusions Increased mobility during childhood is independently associated with adverse health status. At 18, the relationships between residential mobility and self-reported health outcomes, psychological wellbeing and some health behaviours were significant; however, by 36 findings were no longer significant. Directly measured health variables, at 18 and 36 , do not appear to be associated with childhood mobility.

\section{LIFECOURSE PREDICTORS OF ADULT PARENCHYMAL BREAST TISSUE DENSITY: RESULTS FROM THE NEWCASTLE THOUSAND FAMILIES STUDY}

\section{doi:10.1136/jech.2010.120956.42}

${ }^{1} \mathrm{M}$ S Pearce, ${ }^{1} \mathrm{P}$ W G Tennant, ${ }^{2} \mathrm{~T}$ Pollard, ${ }^{3} \mathrm{~L}$ Mclean, ${ }^{3} \mathrm{~B}$ Kaye, ${ }^{4} \mathrm{~L}$ Parker. ${ }^{1}$ Newcastle University, Newcastle-upon- Tyne, UK; ${ }^{2}$ Durham University, Durham, UK; ${ }^{3}$ Newcastleupon- Tyne NHS Foundation Trust, Newcastle-upon- Tyne, UK; ${ }^{4}$ Dalhousie University and Cancer Care Nova Scotia, Halifax, Canada

Objective To investigate the relative influences of factors acting throughout life on breast tissue density at age 49-58 years.

Design Follow-up of the Newcastle Thousand Families birth cohort study.

Setting In 1947, all 1142 babies born in May and June to mothers resident in the city of Newcastle-upon- Tyne were recruited into the Newcastle Thousand Families birth cohort. This study details a sample of women from this cohort who returned for follow-up 49+ years later, and is therefore drawn from throughout the UK and beyond.

Participants At age 50, 574 study members returned a self-completion questionnaire. The 307 surviving women who returned questionnaires at age 50 were sent a questionnaire asking for details of mammographic screening and for details of their reproductive and contraceptive history. 199 women who gave access to their previous mammograms and had completed both questionnaires were included in this analysis.

Main outcome measures Breast tissue density patterns were coded into Wolfe categories $(\mathrm{N} 1=$ lowest risk, $\mathrm{P} 1=$ low risk, $\mathrm{P} 2=$ high risk, $\mathrm{PY}=$ highest risk). This was analysed, by ordinal logistic regression, in relation to a range of variables at different stages of life, including birthweight, gestational age, duration breast fed, age at menarche, gravidity, age at first pregnancy, menopausal status, breast feeding history, hormonal contraceptive history, use of hormone replacement therapy, cigarette smoking history, alcohol consumption, height, body mass index, physical activity levels, age at scan, and socio-economic status both at birth and in adulthood.

Results Eleven \% ( $\mathrm{n}=22)$ of women were classified in the lowest Wolfe category of risk (N1), 20\% (n=39) as low risk (P1), 48\% $(\mathrm{n}=95)$ as high risk (P2), and $22 \%(\mathrm{n}=43)$ as being in the highest category of risk (PY). Increased standardised birthweight (adjusted odds ratio, aOR 1.42 (95\% CI 1.08 to 1.87$), \mathrm{p}=0.01)$ and not having entered the menopause $(\mathrm{aOR}$, compared to perimenopausal women 3.99 (95\% CI 1.78 to 8.97$), p=0.001$ ) were both significant independent predictors of being in a higher density group. In contrast, increasing body mass index was independently predictive of being in a lower density group ( $\mathrm{aOR} 0.85$ (0.80 to 0.91), $\mathrm{p}<0.001$ ) Conclusions After adjustment for factors acting throughout life, this study identified a significant association between increased birthweight, standardised for sex and gestational age, and increased breast tissue density in adulthood. This observation is consistent with previous research suggesting that heavier babies have an increased risk of breast cancer in later life.

\section{ADJUSTING MISCLASSIFICATION OF OUTCOME IN CASE- CONTROL STUDIES}

doi:10.1136/jech.2010.120956.43

${ }^{1} \mathrm{R}$ Gilbert, ${ }^{1} \mathrm{R}$ M Martin, ${ }^{1} \mathrm{~J}$ A Lane, ${ }^{2} \mathrm{D}$ E Neal, ${ }^{3} \mathrm{~F}$ Hamdy, ${ }^{1} \mathrm{~J}$ Donovan, ${ }^{1} \mathrm{C}$ Metcalfe. ${ }^{1}$ Department of Social Medicine, University of Bristol, Bristol, UK; ${ }^{2}$ Department of Oncology, University of Cambridge, Cambridge, UK; ${ }^{3}$ Nuffield Department of Surgery, University of Oxford, Oxford, UK

Objective Misclassification of outcome may cause biased estimation for associations of potential risk factors with important diseases. For example, case-control studies of localised prostate cancer frequently measure blood levels of circulating prostate specific antigen (PSA) in healthy men and biopsy those with an elevated level. Inevitably, some men with prostate cancer will be misclassified as controls, either because they do not have an elevated PSA level or because cancer was not detected at biopsy. This misclassification may be differential if the risk factor itself influences PSA level.

Design We reviewed the literature for methods that correct for non-differential and differential misclassification of outcome in case-control studies. We apply these methods to estimating the association between two established risk factors and prostate cancer: family history and diabetes. We use published data on prostate cancer risk in men with low PSA levels to inform our estimates of the amount of misclassification in our data.

Results Potential approaches range from simple sensitivity analyses to probabilistic sensitivity modelling and Bayesian models, incorporating estimates of sensitivity and specificity. Simple sensitivity analyses recalculate cell frequencies accordingly to produce corrected odds ratios (OR). One accurate estimate of sensitivity and specificity can be used to produce a "corrected" effect-estimate, or a range of values can be used as a sensitivity analysis to assess the direction and magnitude of potential bias. Probabilistic and Bayesian methods incorporate uncertainty in the estimates of sensitivity and specificity as probability distributions so producing a frequency distribution of corrected results from which a median corrected estimate can be presented. Using varying estimates of sensitivity (fixing specificity $100 \%$, feasible in the current example), the direction of the association between family history and prostate cancer (assuming non-differential misclassification) did not change, although the OR increased. The magnitude and direction of the association between diabetes and prostate cancer (assuming differential misclassification) becomes increasingly inverse when the sensitivity in the exposed group is greater than in the non-exposed group. When the sensitivity in the exposed group is smaller than in the non-exposed group, the OR increased, potentially even changing the direction of the association.

Conclusion Correction for misclassification of disease allows presentation of results that incorporate estimates of systematic error due to misclassification bias. That such misclassification may change both the magnitude and direction of an association is demonstrated in real data. Careful consideration is required as to the objective of applying these methods. 